\title{
Angle-dependence of the levitation force from a frustum-shaped magnet and recessed superconducting bulk
}

\author{
James G. Storey, Member, IEEE, and Rodney A. Badcock, Senior Member, IEEE
}

\begin{abstract}
The widespread application of superconducting magnetic levitation bearings is limited by their relatively low stiffness. Recently we investigated a novel thrust bearing geometry comprised of a conical frustum (or truncated cone) shaped permanent magnet levitating inside a matching tapered hole machined into a high-temperature superconductor bulk. This configuration was found to produce superior restoring forces and stiffness compared to the conventional cylindrical magnet and superconductor arrangement. Here, using $H$-formulation finiteelement simulations, we evaluate the angle-dependence of the frustum on the levitation force. We find that the optimal angle is not universal, but depends on the mode of displacement as well as the frustum dimensions. Correlations with the incident magnetic flux are identified for estimating the angle best suited to the operating regime of the bearing.
\end{abstract}

Index Terms-Conical frustum, HTS bulks, levitation force, superconducting magnetic bearing, H-formulation.

\section{INTRODUCTION}

$\mathbf{O}$ FFERING low losses and passive stability, hightemperature superconductor magnetic levitation bearings [1] have the potential to usher in new classes of ultra-highspeed rotating machines [2], [3], [4], [5], [6]. However, this potential is yet to be realised due to their characteristically low stiffness [7], [8], [9]. Higher stiffness can be provided by augmenting the superconducting bearing with an active magnet bearing [10], [11]. But such hybrid approaches come with the cost, complexity and weight of the associated control system.

Recently, we showed that a levitation bearing based on a conical frustum shaped magnet recessed inside a superconducting bulk, similar to that shown in Fig. 1(a), provides improved levitation force and lateral stiffness compared to a typical cylindrical magnet and superconductor arrangement [12]. In this follow-on work, we investigate the angle-dependence of the frustum/recess on levitation force, using previously benchmarked $H$-formulation models, to identify the optimal configuration for this design. The significant advantage of using simulations is the elimination of material variations that are likely to exist between different physical bulks.

\section{Simulation Details}

$H$-formulation simulations implemented in COMSOL Multiphysics [13] were carried out using the method presented

This work was supported by the New Zealand Ministry of Business, Innovation and Employment (MBIE) under Contract RTVU1707. (Corresponding author: James G. Storey.)

The authors are with the Robinson Research Institute, Victoria University of Wellington, Lower Hutt 5046, New Zealand (e-mail: james.storey@vuw.ac.nz). (a)

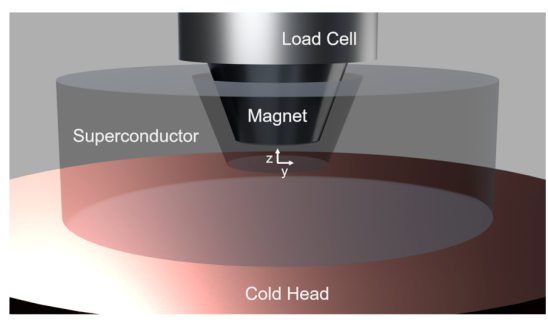

(b)
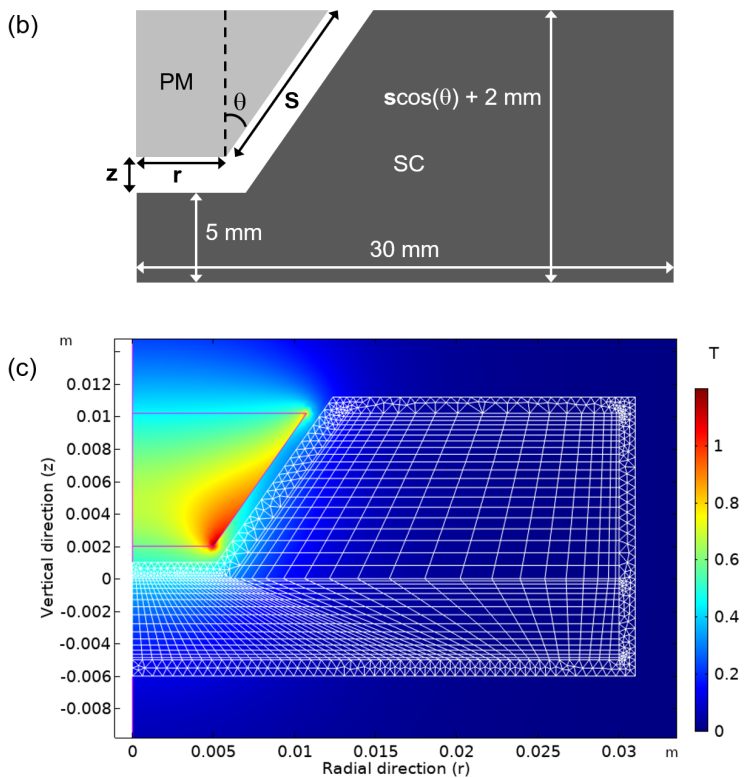

Fig. 1. (a) 3D render of the levitation bearing under investigation. (b) Axisymmetric cross-section (about $r=0$ ) of the bearing geometry. The side length $s$ of the frustum-shaped magnet is fixed at $10 \mathrm{~mm}$, and the minor radius $r$ and angle $\theta$ are varied. (c) 2D axisymmetric finite-element mesh of the superconductor and $1 \mathrm{~mm}$-thick air region superimposed on a color map of the flux density $|B|$ from a magnet with $r=5 \mathrm{~mm}$ and $\theta=35^{\circ}$.

in reference [14]. In this method the field from the magnet is calculated separately using the $A$-formulation and applied as a time-dependent boundary condition on the boundaries of a thin air domain around the superconductor. The key input to the model is the nonlinear resistivity, implemented as a bounded power law [15], [16]

$$
\rho(\mathbf{J})=\frac{\rho_{N S} \cdot \rho_{S C}(\mathbf{J})}{\rho_{N S}+\rho_{S C}(\mathbf{J})}
$$

where the assumed normal-state resistivity is $\rho_{N S}=3.5 \times$ $10^{-6} \Omega \cdot \mathrm{m}, \mathbf{J}$ is the current density, and the resistivity in the 
superconducting state $\rho_{S C}$ is

$$
\rho_{S C}(\mathbf{J})=\frac{E_{c}}{J_{c}}\left|\frac{\mathbf{J}}{J_{c}}\right|^{n-1}
$$

Based on our experiments on YBCO high-temperature superconductor bulks [12] the critical current density was set to $J_{c}=21 \times 10^{8} \mathrm{~A} / \mathrm{m}^{2}$ at the electric field criterion $E_{c}=10^{-4}$ $\mathrm{V} / \mathrm{m}$, and the $n$-value was set to 21 . For simplicity a fieldindependent and spatially homogeneous $J_{c}$ was assumed. To help the calculations converge, a finite resistivity of $1 \Omega \cdot \mathrm{m}$ was applied to the air region [17].

A 2D-axiymmetric representation of the bearing geometry with the magnet $2 \mathrm{~mm}$ above the superconductor is shown in Fig. 1(b). In this work, the minor radius, $r$, and the side length, $s$, are held constant while the angle, $\theta$, that $s$ makes from the vertical is varied. Note that as consequence of these constraints, the vertical thickness of the magnet, $h=s \cos (\theta)$, and the depth of the recess, $h+2 \mathrm{~mm}$, vary with angle. One value of $s(10 \mathrm{~mm})$ and three values of $r(2.5,5,10$ $\mathrm{mm}$ ) were explored. The magnet separation $z$ is defined as the distance between the minor face of the magnet and the bottom of the recess. At their closest separation, the uniform gap between magnet and superconductor is $2 \mathrm{~mm}$, and the top of the superconductor is level with the top of the magnet.

The finite-element mesh is overlaid on a plot of the magnetic flux density from magnet with an angle of $35^{\circ}$ in Fig. 1(c). A remanent flux density of $1.4 \mathrm{~T}$ corresponding to an N50-grade neodymium magnet was used in all cases. Mapped meshes with an arithmetic sequence distribution were applied to the superconductor, while a free triangular mesh was applied to the $1 \mathrm{~mm}$-thick air region. For vertical displacements in this symmetry the relative tolerance was set to $10^{-4}$ and and the absolute tolerance set to $10^{-3}$. For lateral displacements along $y$ a 3D model was created by sweeping the mesh around the $z$-axis, and the relative and absolute tolerances were relaxed to $10^{-3}$ and $10^{-2}$ respectively [14]. The speed of the magnet displacement was set to $1 \mathrm{~mm} / \mathrm{s}$ in all simulations and the force was obtained by integrating $\mathbf{J} \times \mathbf{B}$ over the superconducting domain.

\section{RESULTS}

\section{A. Vertical Displacement}

1) Zero-Field-Cooled: In zero-field-cooling (ZFC) simulations the magnet was moved vertically from an initial position of $z=100 \mathrm{~mm}$, down to $2 \mathrm{~mm}$, and back up to $100 \mathrm{~mm}$. Force vs displacement curves are shown in Figs. 2(a) and (b) for frustum magnets with a minor radius $r$ of $5 \mathrm{~mm}$ and angles $\theta$ ranging from $10^{\circ}$ to $70^{\circ}$. For $\theta$ below about $25^{\circ}$ the curves develop an increasingly convex shape, with the $10^{\circ}$ curve possessing a maximum near $z=6 \mathrm{~mm}$. A similar bellcurve shape was seen previously in our experiments [12] with a frustum/recess angle of $14^{\circ}$. It stems from the average $z$ component of the field normally incident on the side walls of the recess changing sign as it the magnet is lowered into the recess. At higher angles the curves are concave-like and also exhibit reduced hysteresis. The angle-dependent force at $z=2 \mathrm{~mm}$, shown in the inset to Fig. 2(a), roughly follows
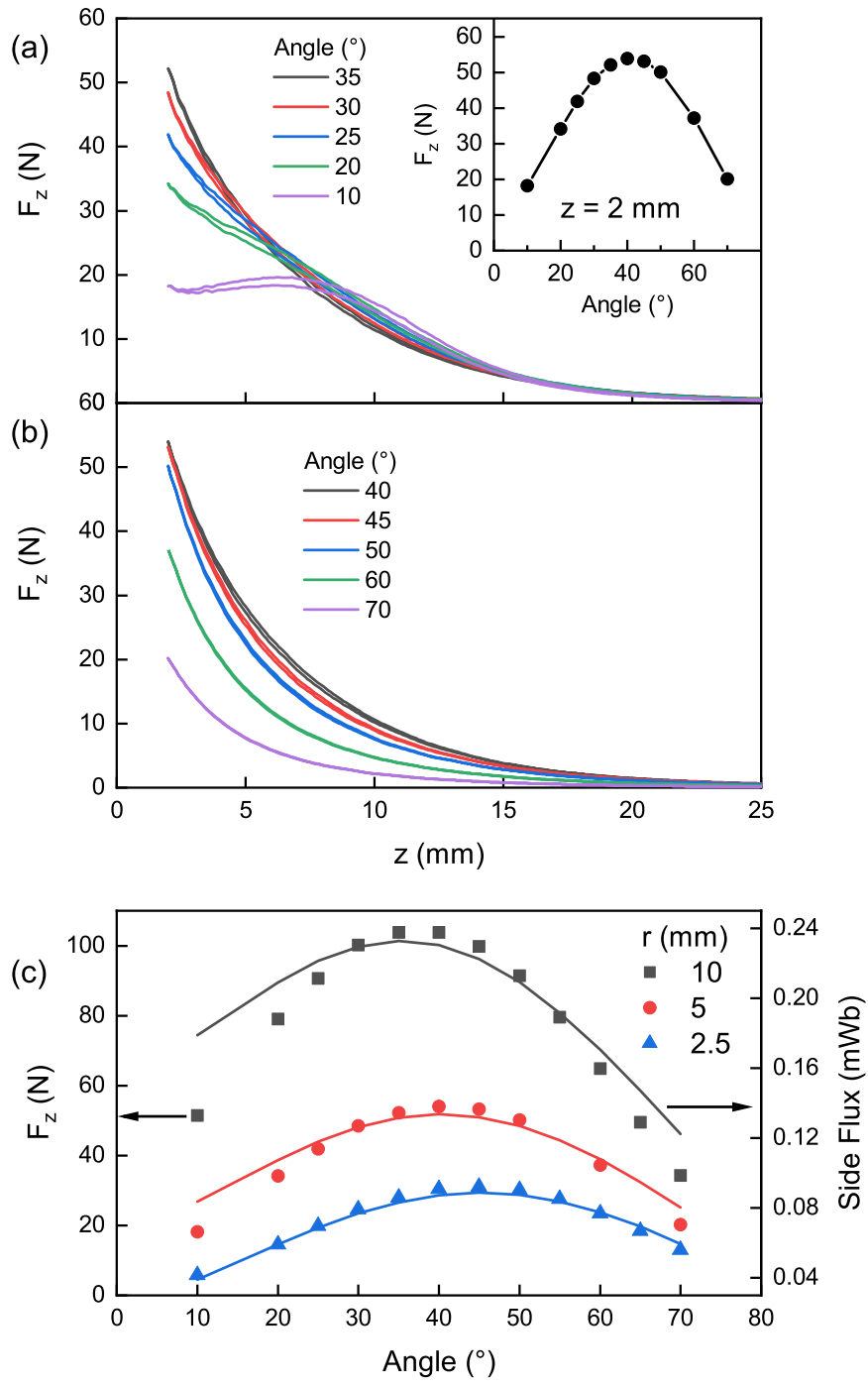

Fig. 2. Zero-field-cooled levitation force vs vertical displacement for $r=5$ $\mathrm{mm}$ and $\theta=10^{\circ}$ to $35^{\circ}$ in (a), and $40^{\circ}$ to $70^{\circ}$ in (b). The inset to (a) shows the angle dependence of the force at $z=2 \mathrm{~mm}$. (c) Angle dependence of the force at $z=2 \mathrm{~mm}$ for $r=5,10$ and $20 \mathrm{~mm}$ (symbols) compared with the flux from the magnet normally incident on the side walls of the recess (lines).

an inverted parabola and is maximal at $\theta=40^{\circ}$. Both the magnitude and optimal angle depend on the size of the minor radius relative to the side length, see Fig. 2(c). The magnitude increases and the optimal angle decreases with increasing $r$, and correlates with the flux from the magnet normally-incident on the side walls of the recess.

2) Field-Cooled: Field-cooled (FC) conditions were simulated by applying the field from the magnet positioned at $z=7 \mathrm{~mm}$ as an initial condition. The magnet was then cycled between $z=2$ and $12 \mathrm{~mm}$. Force-displacement curves for $r=5 \mathrm{~mm}$ and $\theta=10^{\circ}$ to $70^{\circ}$ are shown in Figs. 3(a) and (b). The inset to (a) shows the forces at achieved at the endpoints as a function of angle. The repulsive force at $z=2$ $\mathrm{mm}$ roughly follows an inverted parabolic path, peaking at $35^{\circ}$ which is $5^{\circ}$ lower than the ZFC case.

To help quantify the advantages of this frustum/recess configuration, the forces from a comparative cylindrical magnet 

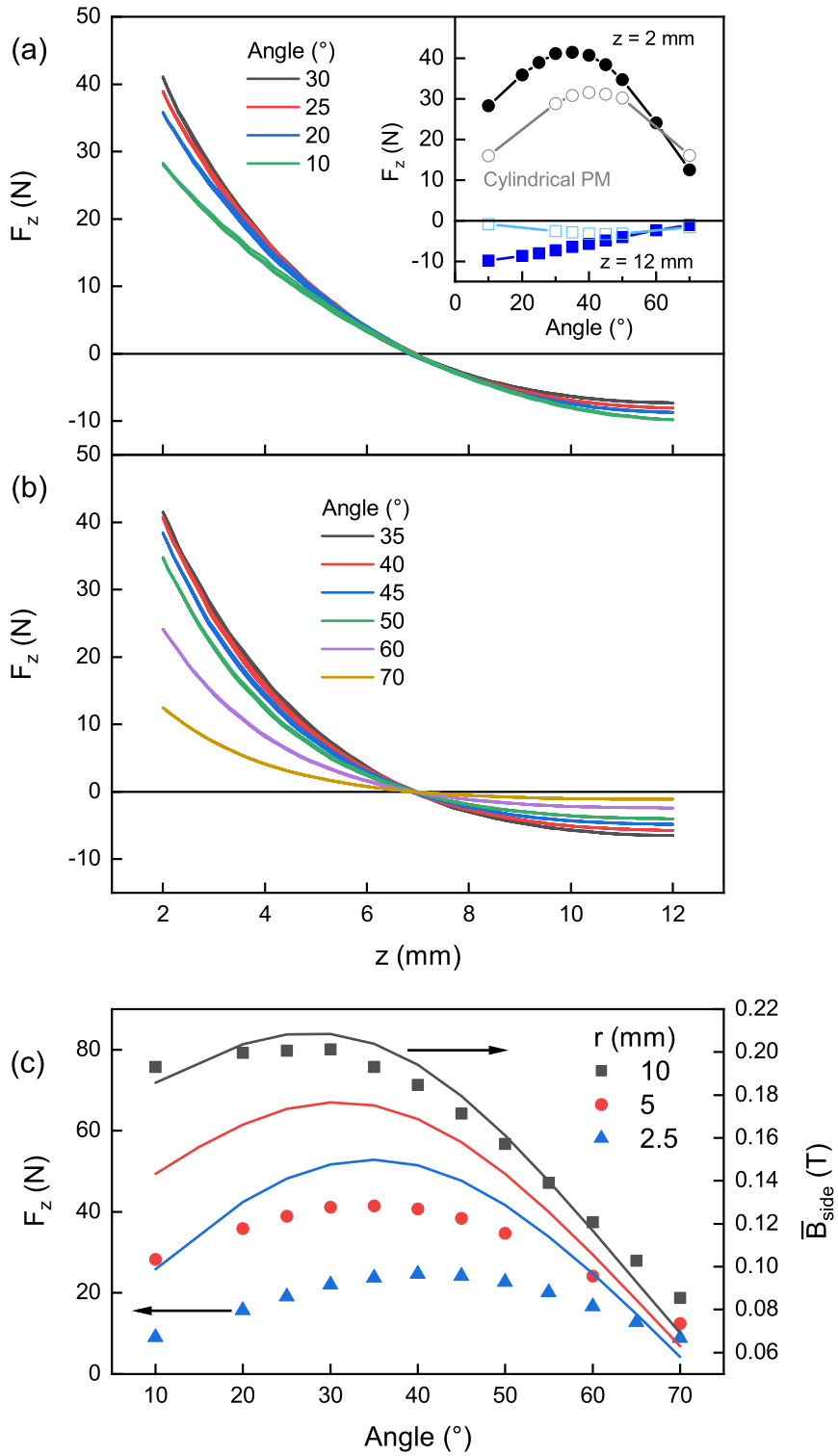

Fig. 3. Field-cooled levitation force vs vertical displacement for $r=5 \mathrm{~mm}$ and $\theta=10^{\circ}$ to $30^{\circ}$ in (a), and $35^{\circ}$ to $70^{\circ}$ in (b). The inset to (a) shows the angle dependence of the force at $z=2$ and $12 \mathrm{~mm}$ (filled symbols) compared with the force from cylindrical magnets levitating above a superconducting disk. (c) Angle dependence of the force at $z=2 \mathrm{~mm}$ for $r=5,10$ and 20 $\mathrm{mm}$ (symbols), and the average field from the magnet normally incident on the side walls of the recess (lines).

levitating above a superconducting disk is also shown. For a given frustum angle, the cylindrical magnet has a diameter equal to the major diameter of the frustum and the same thickness. The superconducting disk is $5 \mathrm{~mm}$ thick with a radius of $30 \mathrm{~mm}$. For angles less than $60^{\circ}$, the frustum/recess provides higher repulsive forces. At $10^{\circ}$ the force is $86 \%$ higher, even though the frustum and cylinder have similar geometries. This indicates that the higher force is due to the sloped walls of the recessed superconductor, rather than the frustum-shaped magnet. Moreover, simulations and experiments using a trapezoidal toroid (which has a sloped hole instead of a recess) produce virtually the same levitation force, confirming that the force originates almost entirely from the
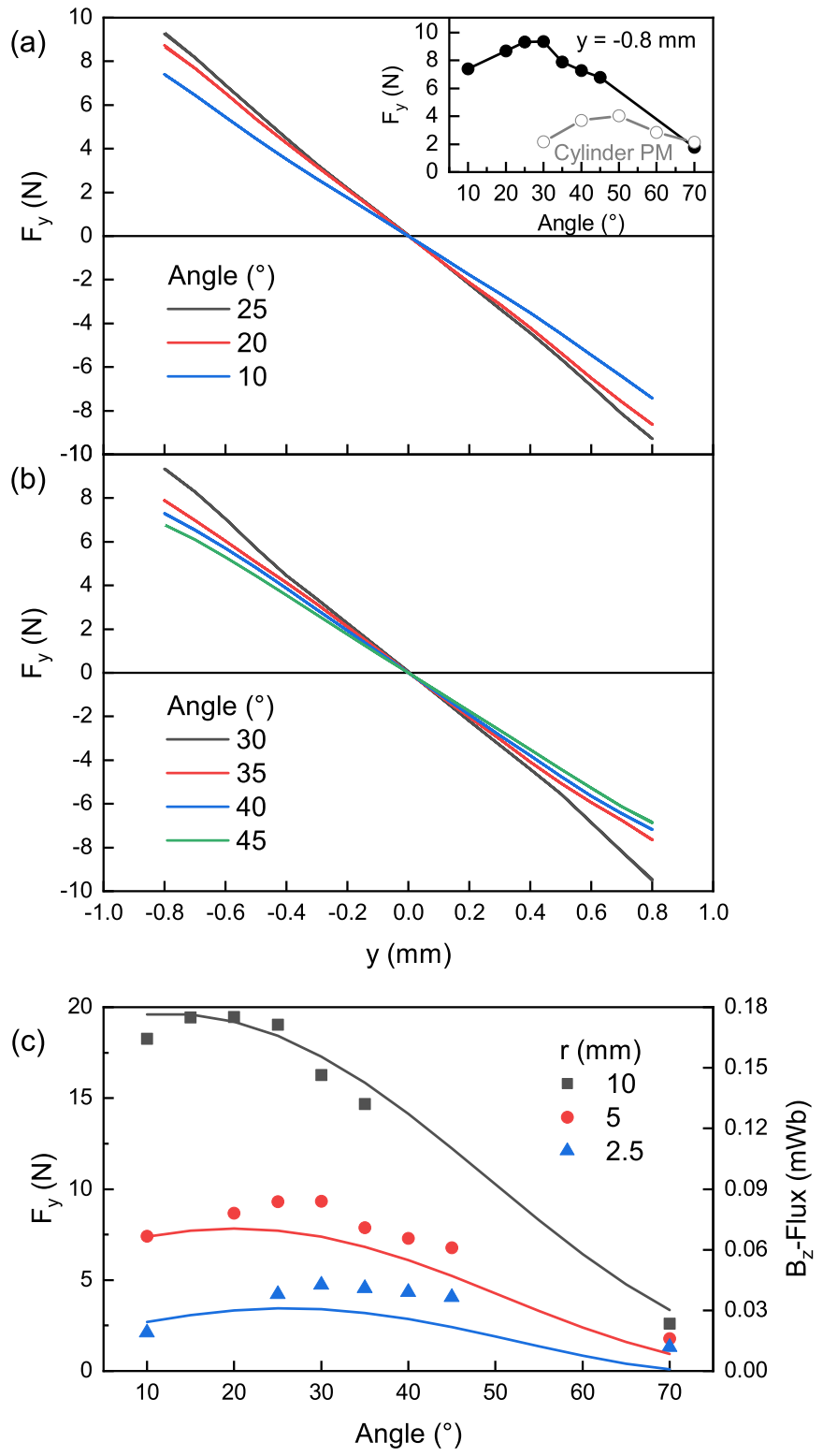

Fig. 4. Restoring force vs lateral displacement for $r=5 \mathrm{~mm}$ and $\theta=10^{\circ}$ to $25^{\circ}$ in (a), and $30^{\circ}$ to $45^{\circ}$ in (b) after field-cooling at $z=2 \mathrm{~mm}$. The inset to (a) shows the angle dependence of the force at $y=-0.8 \mathrm{~mm}$ (filled symbols) compared with the force from cylindrical magnets levitating above a superconducting disk. (c) Angle-dependence of the force at $y=-0.8 \mathrm{~mm}$ for $r=5,10$ and $20 \mathrm{~mm}$ (symbols), and flux from the $z$-component of the field incident on the side walls of the recess (lines).

side walls. For displacements above the initial position, the frustum/recess also delivers higher restoring forces than the cylinder/disc due to the closer average proximity to the magnet afforded by the side walls of the recess.

Like the ZFC case, the magnitude of the force and the angle at which it peaks varies with minor radius, see Fig. 3(c). For $r=2.5$ and $10 \mathrm{~mm}$ the optimal angles are $40^{\circ}$ and $30^{\circ}$ respectively. In this case, the average of the magnetic field component normally incident to the side walls of the recess provides a better predictor of the peak position than the flux. However, it doesn't do so well at predicting the relative magnitudes. The reason for this remains unknown at the time 
of writing, though it is also possible that a different physical quantity might produce a closer match.

\section{B. Lateral Displacement}

Cyclic lateral displacements between $y=-0.8$ and $0.8 \mathrm{~mm}$ were carried out after field-cooling at $z=2 \mathrm{~mm}$. The lateral force along the axis of movement is shown in Figs. 4(a) and (b) for $r=5 \mathrm{~mm}$ and angles from $10^{\circ}$ to $45^{\circ}$. The forcedisplacement curves are approximately linear over this range, and the force at $y=-0.8 \mathrm{~mm}$ (shown in the inset to (a)) peaks between $25^{\circ}$ and $30^{\circ}$ for this value of $r$. The frustum/recess configuration provides over twice the best force achieved from the cylindrical magnet and superconducting disc described above, and does so at a smaller diameter (angle). Again, the maximum force depends on the minor radius. The optimal angles are $30^{\circ}$ and $20^{\circ}$ for $r=10$ and $2.5 \mathrm{~mm}$ respectively. Both the magnitude and angle dependence correlate well with the integral of the $z$-component of the field incident on the side walls of the recess, as shown in Fig. 4(c).

\section{CONCLUSiON}

The optimal frustum/recess angle for producing the highest levitation force is not universal. For a given minor diameter and side length, the optimal angle is largest for zero-fieldcooled vertical displacements, smaller for field-cooled vertical displacements, and smallest for field-cooled lateral displacements. For a given side length and displacement method, the optimal angle decreases as the length of the minor radius increases.

The frustum magnet plus recessed superconductor design provides higher levitation force and stiffness over comparable cylindrical magnet plus superconductor arrangements. Since the force originates from the side walls of the recess, novel through-hole bearings could be realised by boring through the base of the recess.

By comparing the angle-dependence of the force with field maps of the magnets the following empirical relationships were identified. The zero-field cooled vertical force correlates with the flux normally incident on the side walls of the recess. Whereas field-cooled vertical force follows the average of the normally incident field on the side walls. Finally, the field-cooled lateral force correlates with the integral of the $z$ component of the field incident on the side walls. These rules of thumb are useful for quickly estimating the angle best suited to the operating regime of the bearing.

\section{REFERENCES}

[1] J. R. Hull, "Superconducting bearings," Superconductor Science and Technology, vol. 13, no. 2, pp. R1-R15, jan 2000. [Online]. Available: https://doi.org/10.1088/0953-2048/13/2/201

[2] M. Strasik, J. Hull, P. Johnson, J. Mittleider, K. McCrary, C. McIver, and A. Day, "Performance of a conduction-cooled high-temperature superconducting bearing," Materials Science and Engineering: B, vol. 151, no. 3, pp. 195 - 198, 2008. [Online]. Available: http://www.sciencedirect.com/science/article/pii/S0921510708001347

[3] J. R. Hull, M. Strasik, J. A. Mittleider, J. F. Gonder, P. E. Johnson, K. E. McCrary, and C. R. McIver, "High rotational-rate rotor with high-temperature superconducting bearings," IEEE Transactions on Applied Superconductivity, vol. 19, no. 3, pp. 2078-2082, 2009. [Online]. Available: https://doi.org/10.1109/TASC.2009.2017864
[4] J. Xu, R. Chen, H. Hong, X. Yuan, and C. Zhang, "Static characteristics of high-temperature superconductor and hydrodynamic fluid-film compound bearing for rocket engine," IEEE Transactions on Applied Superconductivity, vol. 25, no. 6, p. 3601908, Dec 2015. [Online]. Available: https://doi.org/10.1109/TASC.2015.2477375

[5] S. Kalsi, K. Hamilton, R. Buckley, and R. Badcock, "Superconducting AC homolpolar machines for high-speed applications," Energies, vol. 12, no. 1, p. 86, 2019. [Online]. Available: https://doi.org/10.3390/en12010086

[6] S. Kalsi, R. A. Badcock, K. Hamilton, and J. G. Storey, "Homopolar superconducting AC machines, with HTS dynamo driven field coils, for aerospace applications," IOP Conference Series: Materials Science and Engineering, vol. 756, p. 012028, jun 2020. [Online]. Available: https://doi.org/10.1088/1757-899x/756/1/012028

[7] K. B. Ma, Y. V. Postrekhin, and W. K. Chu, "Superconductor and magnet levitation devices," Review of Scientific Instruments, vol. 74, no. 12, pp. 4989-5017, 2003. [Online]. Available: https://doi.org/10.1063/1.1622973

[8] I. Valiente-Blanco, E. Diez-Jimenez, C. Cristache, M. A. AlvarezValenzuela, and J. L. Perez-Diaz, "Characterization and improvement of axial and radial stiffness of contactless thrust superconducting magnetic bearings," Tribology Letters, vol. 54, no. 3, pp. 213-220, Jun 2014. [Online]. Available: https://doi.org/10.1007/s11249-013-0204-0

[9] A. Cansiz, İrfan Yildizer, and D. T. McGuiness, "Rotor optimization in a superconducting magnetic bearing by using frozen image model and amperian current approximation," Cryogenics, vol. 98, pp. $60-66$, 2019, doi:10.1016/j.cryogenics.2019.01.002.

[10] M. Komori, S. Matsuoka, and S. Fukata, "Evaluations of a hybrid-type superconducting magnetic bearing system," IEEE Transactions on Applied Superconductivity, vol. 6, no. 4, pp. 178-182, Dec 1996. [Online]. Available: https://doi.org/10.1109/77.559341

[11] R. Nicolsky, A. Pereira, R. de Andrade, D. David, J. Santisteban, R. Stephan, A. Ripper, W. Gawalek, T. Habisreuther, and T. Strasser, "Development of hybrid bearing system with thrust superconducting magnetic bearing and radial active electromagnetic bearing," Physica C: Superconductivity, vol. 341348 , pp. $2509-2512$, 2000, proceedings of the International Conference on Materials and Mechanisms of Superconductivity High Temperature Superconductors VI. [Online]. Available: http://www.sciencedirect.com/science/article/pii/S0921453400012983

[12] J. G. Storey, M. Szmigiel, F. Robinson, S. C. Wimbush, and R. A. Badcock, "Stiffness enhancement of a superconducting magnetic bearing using shaped YBCO bulks," IEEE Transactions on Applied Superconductivity, vol. 30, no. 4, p. 600706, 2020.

[13] COMSOL Multiphysics ${ }^{\circledR}$ v. 5.5., http://www.comsol.com. COMSOL $\mathrm{AB}$, Stockholm, Sweden.

[14] L. Quéval, K. Liu, W. Yang, V. M. R. Zermeño, and G. Ma, "Superconducting magnetic bearings simulation using an $\mathrm{H}$-formulation finite element model," Superconductor Science and Technology, vol. 31, no. 8, p. 084001, Jun 2018. [Online]. Available: https://doi.org/10.1088/1361-6668/aac55d

[15] J. Duron, F. Grilli, B. Dutoit, and S. Stavrev, "Modelling the E-J relation of high- $T_{c}$ superconductors in an arbitrary current range," Physica C: Superconductivity, vol. 401, no. 1, pp. 231 - 235, 2004, proceedings of the International Cryogenic Materials Conference: Topical Conference on the VoltageCurrent Relation in Technical Superconductors. [Online]. Available: http://www.sciencedirect.com/science/article/pii/S0921453403015181

[16] A. Morandi, "2D electromagnetic modelling of superconductors," Superconductor Science and Technology, vol. 25, no. 10, p. 104003, sep 2012. [Online]. Available: https://doi.org/10.1088/09532048/25/10/104003

[17] V. Lahtinen, M. Lyly, A. Stenvall, and T. Tarhasaari, "Comparison of three eddy current formulations for superconductor hysteresis loss modelling," Superconductor Science and Technology, vol. 25, no. 11, p. 115001, sep 2012. [Online]. Available: https://doi.org/10.1088/0953$2048 / 25 / 11 / 115001$ 